\title{
NADPH oxidase signaling and cardiac myocyte function
}

\author{
Ashwin Akki, Min Zhang, Colin Murdoch, Alison Brewer \& Ajay M. Shah
}

\section{Introduction}

Reactive oxygen species (ROS) are oxygen-based molecules characterized by high chemical reactivity that play a key role in many physiological and pathophysiological processes. ROS include radicals such as superoxide (O2-radical dot) and hydroxyl (OHradical dot) and non-radicals such as hydrogen peroxide (H2O2). While oxidative stress (where pro-oxidants overwhelm antioxidant capacity, leading to excess ROS levels) is well known to damage macromolecules, membranes and DNA, the tightly regulated production of ROS also has important biological effects by modulating the activity of diverse intracellular signaling pathways involved in cell survival, growth, metabolism and motility (termed 'redox signaling') [1] and [2]. The NADPH oxidase family of enzymes generate O2-radical dot in a highly regulated manner by catalyzing electron transfer from NADPH onto molecular $\mathrm{O} 2$ and are thought to be especially important in redox signaling [3], [4] and [5]. Emerging evidence suggests the involvement of NADPH oxidase-derived ROS in a range of cardiac physiological and pathophysiological processes.

\section{Sources of ROS in the heart}

All cell types within the heart, including cardiomyocytes, endothelial cells, vascular smooth muscle cells (VSMCs), fibroblasts and infiltrating inflammatory cells generate ROS. Potential sources of ROS in these cell types include the mitochondrial electron transport chain, xanthine oxidases, 'uncoupled' nitric oxide synthases, cytochrome P450 and NADPH oxidases. Among these sources, the NADPH oxidases may be considered unique in that they generate ROS in a highly regulated manner whereas ROS are generated as a by-product of enzymatic activity for all the other sources [3], [4] and [5]. Furthermore, NADPH oxidases can stimulate further ROS production from one or more of the above enzymes, thereby being able to act as initiating sources of ROS. O2-radical dot is the first moiety that is generated by NADPH oxidases (or most of the other sources) and can be rapidly dismutated to $\mathrm{H} 2 \mathrm{O} 2$. The biological effects of ROS are expected to depend on the specific moiety generated, its localisation and the relative balance between levels generated and the activity of antioxidant mechanisms; most signaling effects of ROS are considered to be mediated by $\mathrm{H} 2 \mathrm{O} 2$ which is more stable and diffusible than $\mathrm{O} 2$-radical dot.

\section{NADPH oxidase isoforms}

The prototypic NADPH oxidase is a multi-subunit enzyme complex that was first identified in neutrophils, where it is involved in non-specific host defence against microbes during phagocytosis. In the last decade, five oxidase isoforms each encoded by a separate gene and with distinct tissue distribution have been identified [3] and [5]. These isoforms are distinguished by the presence of distinct catalytic subunits, Nox1-Nox5, which mediate the electron transfer process; Nox2 (or gp91phox) is the original neutrophil isoform. In addition to the core catalytic Nox subunit, the enzymatic activity of the oxidase depends on additional protein subunits, which vary according to the isoform. Nox1-Nox4 - each associates with a smaller p22phox subunit, which provides stability to the complex. Nox1 and Nox2 require the association of additional protein subunits in order to become fully activated. In the case of Nox2, these are 4 cytosolic regulatory subunits, namely p47phox, p67phox, p40phox and Rac1 or Rac2, whereas Nox1 associates with Rac1 and homologues of p47phox and p67phox named NoxO1 and NoxA1 respectively. The Nox catalytic subunits contain 5-6 transmembrane domains, binding sites for NADPH and FAD (flavin adenine dinucleotide), and two haem domains that can undergo cyclical reduction and oxidation. Nox5 differs from Nox1-4 in having an additional calmodulin-like cytoplasmic domain that contains four binding sites for calcium, allowing its activation by cytosolic calcium [6]. Two further, more complex enzymes called the Duoxes contain domains homologous to the Noxs and a calcium-binding domain, but in addition also have a "peroxidase-homology" domain [3] and [5]; there is no evidence so far that these have important roles in the cardiovascular system and they are not considered further here. 
In the cardiovascular system, Nox1 is expressed mainly in VSMCs [7]. Nox2 is expressed in endothelial cells [8] and [9], cardiomyocytes [10], [11] and [12], fibroblasts [13] and some VSMCs [14]. Nox4 is expressed in endothelial cells [15], VSMCs [16], cardiomyocytes [17] and [18] and fibroblasts [19]. Nox3 is not expressed in cardiovascular cells while Nox 5 has been reported in human endothelial cells and smooth muscle cells but is not found in rodents [20] and [21]. The cytosolic components of the classical NADPH oxidase - i.e., p47phox, p67phox, p40phox and Rac1 have been detected at both mRNA and protein levels in most cardiovascular cells except for p67phox which could not be detected in cultured VSMCs [22]. Fig. 1 illustrates the main Nox isoforms expressed in the myocardium.

Fig. 1. - Schematic diagram illustrating the different Nox isoforms expressed within the heart. Cardiomyocytes, endothelial cells and fibroblasts all express Nox 2 and Nox4. Nox 2 is also expressed in inflammatory cells while human endothelial cells may express Nox5. The cells that may express eNOS, iNOS and nNOS are also shown.

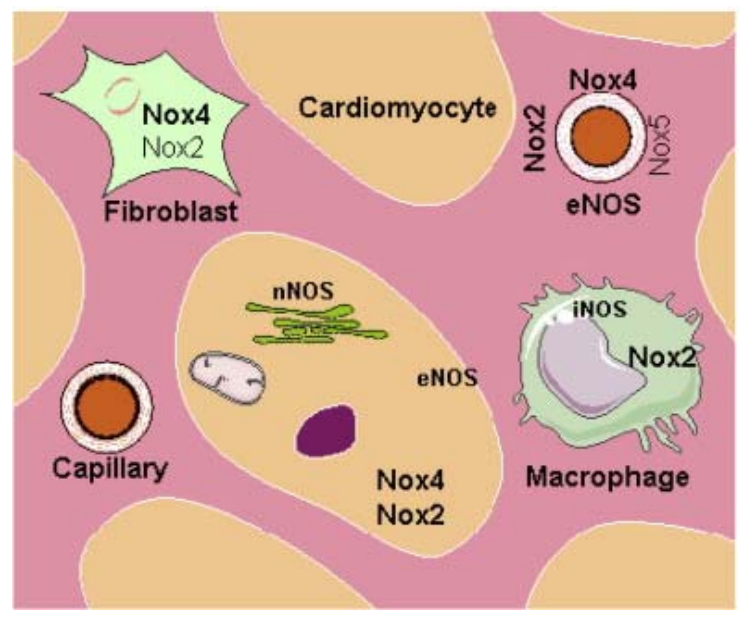

\section{NADPH oxidase activation and downstream signaling}

A wide range of agonists and stimuli can activate NADPH oxidases in cardiovascular cells [22], [23] and [24]. These include (i) G-protein coupled receptor agonists (GPCRs) such as angiotensin II, endothelin-1 and $\alpha$-adrenergic agonists; (ii) growth factors, e.g. thrombin and vascular endothelial growth factor (VEGF); (iii) cytokines, e.g. tumor necrosis factor- $\alpha$ (TNF- $\alpha$ ) and transforming growth factor $\beta$ (TGF $\beta$ ); (iv) mechanical forces; (v) metabolic factors, e.g. insulin, glucose and free fatty acids, glycated proteins; and (vi) hypoxia-reoxygenation. The details of the specific isoform(s) activated by these stimuli remain to be fully established and may vary according to cell type. The majority of information to date is derived from analyses of Nox1 activation in VSMCs [23] and [25] and Nox2 activation in endothelial cells [26], [27], [28] and [29], with only a handful of studies examining Nox isoform-specific activation in cardiomyocytes [11] and [30].

The activation of Nox 1 or Nox2 is a complex process that involves multiple post-translational modifications of oxidase regulatory subunits (notably p47phox and Rac), which facilitate their translocation and binding to the Noxp22phox heterodimer, followed by activation of electron transfer (reviewed in refs. [4, 5]). The complexity of this highly regulated stimulus-initiated process would appear to be well suited for involvement in cell signaling pathways. Recent studies suggest that such cell signaling may be facilitated by the assembly of signaling complexes including receptors, NADPH oxidase components and downstream signaling targets in cellular microdomains such as endosomes [31] and [32]. In marked contrast to Nox1 and Nox2, Nox4 is reported to be constitutively active and not to require association with any known cytosolic regulatory subunits for its activation [33] and [34]. Nevertheless, it has been reported to specifically modify signal transduction induced by insulin and epidermal growth factor [35], [36] and [37] and a recent study suggests that this may involve specific modulation of receptor activation and trafficking at the level of the endoplasmic reticulum [35]. In addition to the above acute mechanisms, changes in expression level of the different proteins required for the fully activated Nox complex (e.g. through transcriptional pathways) may also influence enzyme activity [4] and [5]. All NADPH oxidases require NADPH as an electron donor and it has recently been suggested that NADPH availability could also influence oxidase activity in some settings [38]. 
In this regard, the activity of glucose-6-phosphate dehydrogenase could be important since this is a key determinant of NADPH levels in the cell.

Nox-derived ROS may mediate their effects through diverse downstream signaling pathways including: (i) the activation of redox-sensitive kinases [such as protein kinases B and C, members of mitogen activated protein kinase (MAPK) family and Janus kinase (JAK)] either indirectly via inhibition of protein tyrosine phosphatases or directly; (ii) altering the activity of redox-sensitive transcription factors such as activator protein-1 (AP-1), nuclear factor-kappa B (NF-KB), hypoxia-inducible factor-1 (HIF-1) and signal transducer and activator of transcription (STAT); and (iii) direct effect on enzymes [e.g. matrix metalloproteinases (MMPs)], ion channels or receptors. This topic has been comprehensively covered in recent reviews [4], [5], [24], [25] and [39], and in the current article we limit our discussion to effects in the myocardium.

\section{Effects of NADPH oxidase on calcium signaling}

The regulation of $\mathrm{Ca} 2+$ in cardiac myocytes is centrally important not only in excitation-contraction coupling but also in many other processes such as the regulation of gene expression and cellular energetics. ROS are recognized to be capable of influencing cellular Ca2+ regulation at several levels, notably via redox modifications of key amino acid residues involved in the function and gating properties of intracellular and plasma membrane ion channels and transporters - e.g., L-type channels, the $\mathrm{Na}+\mathrm{Ca} 2+$ exchanger, the sarcoplasmic reticulum (SR) ATPase (SERCA) and the ryanodine receptor [40] and [41]. Recent studies have started to address the role of NADPH oxidase-derived ROS in these effects.

It has been reported that ryanodine receptor-mediated $\mathrm{Ca} 2+$-induced $\mathrm{Ca} 2+$ release in rat cardiac myocytes is inhibited by an endogenous NADH oxidase activity in the SR, although the molecular nature of this oxidase was not established [42]. In contrast to this study, Sanchez et al. [43] reported the presence of Nox2 NADPH oxidase activity in canine cardiac SR and showed that oxidase activation increased S-glutathionylation of ryanodine receptors and hence SR Ca2+ release - effects which were abrogated by apocynin (a purported Nox inhibitor, but which may act as a non-selective antioxidant). The same group also showed that oxidase activity and the effects on SR Ca2+ release were augmented by tachycardia [44]. O2-radical dot production by NADPH oxidase on the SR of bovine coronary artery smooth muscle cells has also been shown to regulate calcium-induced calcium release [45]. In isolated cardiac myocytes, plasma L-type Ca2+ channel open-state probability was reportedly increased by endothelin-1 together with enhanced NADPH oxidase activity, effects which were abolished in cardiomyocytes pre-treated with a specific NADPH oxidase inhibitor, gp91ds-tat [46]. These studies suggest that NADPH oxidases may acutely regulate at least two channels directly involved in intracellular Ca2+ homeostasis, i.e. the L-type Ca2+ channel and the ryanodine receptor.

NADPH oxidases have also been reported to modulate the activities of other ion channels. In streptozotocin-induced diabetes in rats, membrane $\mathrm{K}+$ currents were attenuated secondary to increased angiotensin II-induced NADPH oxidase activation [47]. Angiotensin II-induced swelling-activated $\mathrm{Cl}$ - current in rabbit ventricular myocytes involved NADPH oxidase activation, and was inhibited by a range of inhibitors including gp91ds-tat [48]. Interestingly, ROS generated by NADPH oxidase may also modulate the expression of ion channels. Zhou et al. [49] found that Kv4.3 channel mRNA was destabilized by angiotensin II- or stretch-activated NADPH oxidase in neonatal rat ventricular myocytes, while Tsai et al. [50] reported that expression of the L-type channel alpha1c subunit was increased by angiotensin II through a protein kinase C/NADPH oxidase pathway in HL-1 cells. In H9c2 myocytes or freshly isolated myocytes, angiotensin II decreased $\mathrm{Na}+$ channel (scn5a) mRNA abundance via NADPH oxidase and NF-KB activation [51]. All the above angiotensin II-induced effects are likely to involve Nox2 oxidase activation.

\section{Cardiac hypertrophy}

Cardiac hypertrophy is an independent risk factor for the development of heart failure and a major cause of morbidity and mortality. Multiple intracellular signaling pathways are implicated in the genesis of cardiac hypertrophy, including various redox-signaling pathways [52]. Studies of hypertrophy in isolated cultured 
cardiomyocytes found an important role for intracellular ROS generation in response to $\alpha$-adrenergic agonists [12], angiotensin II [53], endothelin-1 [54] and TNF- $\alpha$ [53], and Xiao et al. [12] provided pharmacological evidence that $\alpha$ adrenergic-induced hypertrophy involved NADPH oxidase. Work from our group found that NADPH oxidase activity was enhanced in pressure-overload cardiac hypertrophy in association with increased Nox2 oxidase expression in both cardiomyocytes and endothelial cells, and that this paralleled the activation of the redox-sensitive kinases ERK1/2, ERK5 and p38MAPK [55]. It has also been shown that NADPH oxidase subunit expression and activity are increased in the myocardium of human failing hearts [56], [57] and [58], suggesting the importance of this pathway in humans.

Definitive evidence for the involvement of NADPH oxidases in pathological hypertrophy came from experiments in genetically modified mice. We found that the in vivo cardiac hypertrophic response to short-term subpressor angiotensin II infusion was markedly inhibited in Nox2-/- mice compared to wild-type, and that this was associated with a blunting of the increase in molecular markers of hypertrophy such as ANF mRNA [10]. In line with this finding, angiotensin II-induced hypertrophy of isolated cardiomyocytes was subsequently also definitively shown to involve the activation of Nox2 oxidase [11]. Further strong evidence for an involvement of Nox 2 in the in vivo hypertrophic response to angiotensin II came from a study in mice with conditional cardiac-specific knockout of Rac1, which had blunted hypertrophy as well as reduced myocardial Nox2 oxidase activity [59]. On the other hand, mice with cardiacspecific overexpression of Rac1 displayed a cardiomyopathic phenotype characterized by hypertrophy and dilatation, although it should be noted that Rac1 may have multiple additional effects apart from acting as a co-factor for NADPH oxidase activation [60]. Nevertheless, these data are in agreement with prior studies where transfection of cultured neonatal cardiomyocytes with a constitutively active Rac1 mutant induced hypertrophy whereas a dominant-negative Rac1 mutant inhibited the hypertrophic response to phenylephrine [61] and [62].

In contrast to angiotensin II-induced hypertrophy, the hypertrophic response to aortic banding was found to be similar in Nox2 knockout mice and wild-type controls both by our group [17] and by Maytin et al. [63]. However, we found that aortic banding induced similar increases in myocardial NADPH oxidase activity in wild-type and Nox2-/mice, which could be attributed to increased expression of Nox4 mRNA and protein levels [17]. Furthermore, chronic treatment with the antioxidant $\mathrm{N}$-acetylcysteine attenuated hypertrophy in both groups of mice. These results indicate that Nox2 is not essential for the development of pressure-overload hypertrophy and that other sources of ROS (including possibly Nox4 oxidase) may be involved in the response to chronic pressure-overload.

The pro-hypertrophic signaling pathways triggered by NADPH oxidase-derived O2-radical dot in cardiomyocytes remain to be fully defined. Nonetheless, current evidence supports the involvement of the following pathways: (i) ERK-1/2 [12] and [64] and the small GTPase Ras [65] in response to $\alpha$-adrenergic agonist stimulation and angiotensin II [59] and [66], (ii) MAPKs in pressure-overload hypertrophy [55] and (iii) NF-KB and apoptosis-signal-regulated kinase 1 (ASK-1) in response to angiotensin II infusion [59]. The involvement of ASK-1 is further supported by the finding that the in vivo hypertrophic response to angiotensin II is attenuated in ASK-1 knockout mice [67] and that the hypertrophic response to GPCR agonists is inhibited in cultured cardiomyocytes expressing a dominant-negative mutant of ASK-1 [54]. ASK-1 is upstream of p38MAPK and JNK in the MAPK signaling cascade, and both of these have been shown to be activated by NADPH oxidases [68].

\section{Apoptosis}

The loss of cardiomyocytes through apoptosis adversely affects overall contractile function and the progression to heart failure in the setting of chronic pressure-overload or adverse remodeling post-MI [69] and [70]. Oxidative stress is well known to induce cardiomyocyte apoptosis either indirectly, through damage to DNA, lipids and proteins, or more directly via the activation of pro-apoptotic signaling molecules such as ASK-1, JNK, ERK1/2 and p38 MAPK [68]. A number of studies have investigated the role of NADPH oxidase-derived ROS in cardiomyocyte apoptosis. Apocynin was reported to abolish the apoptotic response to angiotensin II in rat embryonal cardiomyocytes, suggesting the involvement of NADPH oxidase in this process, although as noted previously this inhibitor is now recognized to have non-specific antioxidant activity [71]. In vivo apocynin treatment also blunted the 
rise in myocardial NADPH oxidase activity, O2-radical dot production, cardiomyocyte apoptosis and elevation of proapoptotic Bax protein in a rabbit model of $\mathrm{MI}$, in association with an improvement in ventricular function [72]. A recent study by Mehta et al. [73] demonstrated that viral infection of cultured mouse cardiomyocytes with an active form of TGF $\beta 1$ (TGFB1ACT) reduced apoptosis following hypoxia-reoxygenation together with a reduction in NADPH oxidase activity, O2-radical dot production and NF-KB expression. These findings were confirmed in vivo in rats subjected to intramyocardial injection of TGF $\beta 1$ ACT followed by left coronary artery ligation.

The possible mechanisms underlying a pro-apoptotic role of Nox-derived ROS remain unclear. It has been proposed that angiotensin II-induced apoptosis is mediated, in part, via NADPH oxidase-dependent peroxynitrite formation and consequent DNA damage [74]. Likewise, TNF $\alpha$-induced apoptosis of cultured mouse cardiomyocytes was reported to involve an NOS/NADPH oxidase-dependent, peroxynitrite-mediated signaling pathway [75]. In neonatal rat cardiomyocytes, aldosterone was shown to induce apoptosis by activating NADPH oxidase-mediated O2-radical dot production and ASK-1 [76]. Collectively, these studies suggest that NADPH oxidase-derived ROS may play a role in cardiomyocyte apoptosis in response to a number of pathogenic stimuli.

\section{Contractile dysfunction}

The decline in cardiac contractile function heralds the onset of a 'decompensated phase' in the evolution of heart failure. Multiple mechanisms contribute to myocardial dysfunction including altered excitation-contraction coupling, impaired myofilament calcium responsiveness, mitochondrial dysfunction and energy deficit, extracellular matrix remodeling and the loss of myocytes. A significant body of evidence implicates oxidative stress in the development of contractile dysfunction through one or more of these mechanisms [77], [78], [79], [80], [81], [82], [83] and [84], but the potential role of NADPH oxidase-derived ROS remains to be established.

The preceding discussion on the effects of NADPH oxidase-derived ROS on calcium signaling clearly suggests their potential to influence contractile function. A few recent studies provide some direct evidence in support of this possibility. In work from our laboratory, we studied the possible contribution of Nox2 oxidase-derived ROS to contractile dysfunction in pressure-overload hypertrophy [85]. We found that Nox2-/- mice subjected to aortic banding had better contractile function than wild-types as assessed by echocardiography or ex vivo pressurevolume analyses despite having a similar degree of hypertrophy. This was confirmed at the cellular level in isolated ventricular myocytes from these animals and it was also shown that contractile function could be acutely improved in the wild-type group by treatment with $\mathrm{N}$-acetylcysteine, suggesting that at least part of the defect was the result of acute changes in contractile properties (as opposed to altered expression of relevant proteins). Li et al. [86] demonstrated that the contractile defect in cardiomyocytes incubated with angiotensin II was abolished by apocynin in association with evidence of reduced activation of Nox2 oxidase. A similar involvement of NADPH-oxidase-derived ROS in cardiomyocyte dysfunction has been suggested in obesity [86], streptozotocin-induced diabetes [87] and [88], aging [89] and gram-negative sepsis [90]. Nox2 is also involved in post-MI contractile dysfunction as discussed in a subsequent section, although this may be secondary to improved remodeling. Finally, NADPH-oxidase-derived ROS may also influence ventricular function indirectly through the inactivation of nitric oxide, as demonstrated in a guinea pig model of chronic pressure-overload [91].

\section{Interstitial fibrosis}

Interstitial and perivascular fibrosis are characteristic features of pathological ventricular hypertrophy [52], the physiological consequences of which include - (i) increased myocardial stiffness resulting in greater diastolic filling pressures (i.e. diastolic dysfunction), (ii) disruption of myofibre organisation contributing to systolic dysfunction, (iii) altered myocardial electrical properties leading to arrhythmias and (iv) increased diffusion distances between coronary vessels and cardiomyocytes contributing to relative ischemia in the absence of overt coronary artery disease. A large body of evidence indicates that oxidative stress may be pro-fibrotic, while NADPH oxidase-derived ROS have been implicated in both interstitial and perivascular fibrosis in pathological cardiac hypertrophy (as indeed seems to be the case in peripheral blood vessels [13] and [22]). 
Work from our laboratory revealed that interstitial cardiac fibrosis was significantly attenuated in Nox2-/- mice subjected to either sub-pressor or pressor infusion of angiotensin II for 2 weeks, in association with reduction in NADPH oxidase activity [10], [17] and [92]. Similarly, angiotensin II-induced interstitial fibrosis was inhibited in conditional Rac1 knockout mice [59] and ASK-1 knockout mice [67]. Johar et al. [92] further showed that interstitial fibrosis in response to chronic infusion of the mineralocorticoid receptor (MR) agonist aldosterone in combination with a salt diet was dependent on Nox2 oxidase since it was inhibited in Nox2-/- mice. Furthermore, the effects of angiotensin II could also be partially blocked by an MR receptor antagonist, spironolactone. These data are in line with the observation that interstitial cardiac fibrosis in MR-dependent hypertension in rats was associated with an increase in myocardial oxidative stress and Nox2 expression, [93] effects which were inhibited by chronic treatment with apocynin [94]. In addition, the extensive perivascular fibrosis observed following angiotensin II infusion in transgenic mRen 2 rats was associated with chronically elevated tissue levels of angiotensin II, increased NADPH oxidase activity and 02-radical dot production [95]. Nox2 appears to have similar pro-fibrotic effects in mice subjected to chronic pressure-overload since Nox2-/- mice have significantly less interstitial fibrosis despite a similar degree of hypertrophy to wild-types [85].

Investigation into the mechanisms underlying angiotensin II-induced Nox2-dependent cardiac interstitial fibrosis revealed a marked upregulation of the profibrotic cytokine connective tissue growth factor (CTGF) and profibrotic genes (procollagen-1, procollagen-3 and fibronectin) in wild-type but not Nox2-/- mice. [92]. In addition, the activation of NF-KB and matrix metalloproteinase-2 (MMP-2) were also found to be Nox2-dependent in this model. A similar Nox-dependent MMP activation in vascular smooth muscle has been shown in response to stretch and angiotensin II [96] and [97]. In ASK1 knockout mice subjected to angiotensin II infusion, the attenuation of interstitial fibrosis was associated with reduced p38MAPK and JNK activation [67], although the anti-fibrotic effects may be secondary to reduced cardiomyocyte hypertrophy. In contrast to the above studies, it should be noted that studies in isolated cultured cardiac fibroblasts have implicated Nox4 NADPH oxidase in fibroblast proliferation and transformation into myofibroblasts [19]. An explanation for this apparent contradiction could well be the fact that interstitial fibrosis in vivo is a complex process involving interactions among multiple cell types; the evidence in support for a role of Nox 2 in vivo appears strong but its cell-specific contribution and the in vivo role of Nox 4 remain to be established.

\section{Ventricular remodeling after myocardial infarction}

Ventricular remodeling post-MI is characterized by myocyte death and marked inflammation in the infarct and periinfarct areas in the early phase followed by significant hypertrophy, fibrosis and prominent extracellular matrix remodeling in the non-infarcted myocardium in the later phase [98] and [99]. Enhanced oxidative stress post-MI has been consistently described in both clinical and experimental studies [100]. Indeed, a variety of antioxidant approaches could attenuate adverse ventricular remodeling after experimental MI [81] and [101], thus implicating oxidative stress in this pathology.

Fukui et al. [102] were one of the first groups to suggest the involvement of NADPH oxidases in post-MI remodeling. These authors observed elevated Nox2 and p22phox expression levels in the rat myocardium after experimental MI. In a subsequent study, similar findings were reported in post-mortem myocardial samples from humans who had succumbed to acute MI [103]. More direct evidence for the involvement of Nox2 oxidase in adverse remodeling post-MI has come from studies in gene-modified mice. Dorries et al. [104] studied p47phox knockout mice and wildtype littermates subjected to left coronary ligation and found that the oxidase-deficient animals had significantly less remodeling with reduced ventricular dilatation and improved ejection fraction. Furthermore, the p47phox-/- mice had reduced myocyte apoptosis and improved overall survival despite similar infarct sizes to wild-type controls. We found essentially similar results in Nox2-/- mice subjected to experimental MI [105]. Nox2-/- animals had significantly less cavity dilatation and a higher fractional shortening after MI than their wild-type counterparts, and better systolic and diastolic function by in vivo cardiac catheterization. At the cellular level, Nox2-/- mice had less myocyte hypertrophy and reduced interstitial fibrosis in the non-infarcted myocardium, associated with a blunted 
increase in the mRNA expression of ANF, CTGF, procollagen-1 and fibronectin and an attenuation of MMP activation. Taken together, these studies highlight a crucial role of Nox2 oxidase in post-MI remodeling.

\section{Ischemic preconditioning}

Ischemic preconditioning refers to the cardioprotective phenomenon whereby transient episodes of ischemia result in significant protection against myocardial injury induced by subsequent prolonged ischemia [106]. The mechanisms underlying this potent protective effect still remain incompletely understood but inhibition of the mitochondrial permeability transition is increasingly considered to be an important terminal mechanism. Many experimental studies have highlighted the role of redox signaling in the cardioprotection offered by ischemic preconditioning [107], [108] and [109]. While mitochondrial-derived ROS are widely considered to be involved in the pathway, there is also evidence for a signaling role of NADPH oxidase-derived ROS in the proximal pathways of preconditioning. Bell et al. [110] studied ischemic preconditioning in isolated buffer-perfused hearts from mice with a global deficiency of Nox2 (Nox2-/- mice) and matched wild-type mice, and showed that Nox2 knockout mice failed to precondition in response to brief global ischemia. In contrast, hearts of wild-type mice had enhanced NADPH oxidase activity and showed attenuated infarct sizes after preconditioning. However, the myocardium of Nox2-/- mice could still be preconditioned by an adenosine receptor agonist. Subsequently, other groups have reported an involvement of NADPH oxidase-derived ROS in the cardiac preconditioning induced by angiotensin II [111] and [112] and tachycardia or exercise [43] and [44].

Further investigation into the signaling mechanisms involved in the above effects revealed that protein kinase $\mathrm{C}$ was essential for the activation of Nox2 oxidase in ischemic preconditioning [110]. In angiotensin II-dependent preconditioning, NADPH oxidase-induced activation of ASK-1 and p38MAPK were suggested to trigger mitochondrial ROS generation and consequent cardioprotection [111], whilst in tachycardia/exercise-mediated preconditioning NADPH oxidase-induced modulation of SR Ca2+ release was proposed as the mechanism mediating the protective response [44].

\section{Conclusions}

An increasing body of evidence implicates ROS-generating NADPH oxidases in redox signaling in cardiovascular cells and involvement in pathological processes such as cardiac hypertrophy, fibrosis, apoptosis and ventricular remodeling (Fig. 2). In view of the lack of good pharmacological probes, data on the relative importance of different oxidase isoforms is currently best derived using specific molecular approaches. An involvement of Nox2 oxidase in several important cardiac conditions has been quite convincingly demonstrated in studies using mice deficient in Nox2 or other subunits required for its activation, although the precise signaling pathways downstream of the oxidase that are involved require further study. In contrast, very little is yet known about the in vivo roles of Nox4 oxidase. A better understanding of the regulation of these different Nox isoforms and their involvement in specific redox signaling pathways could allow the development of novel therapeutic strategies. 
Fig. 2. - Potential effects of NADPH oxidase-derived ROS in the cardiac myocyte. SR, sarcoplasmic reticulum, MMPs, matrix metalloproteinases; CICR, calcium-induced calcium release.

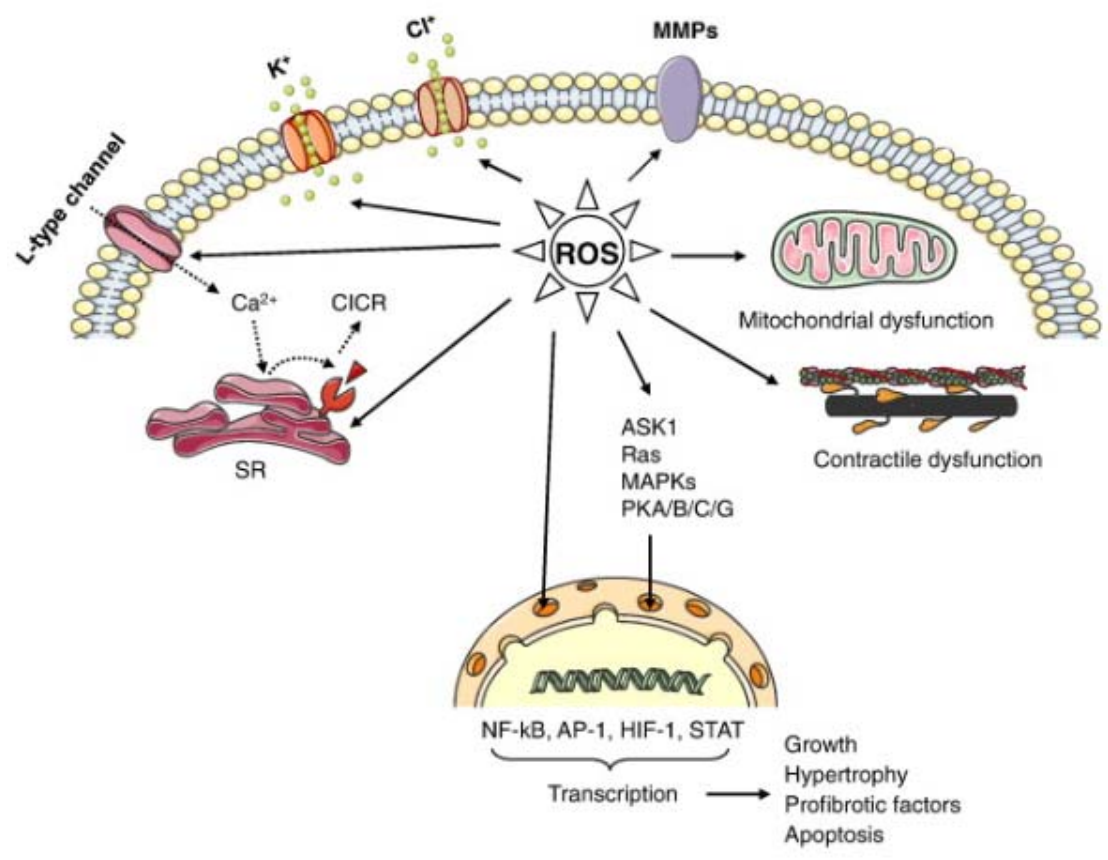

\section{References}

[1] T. Finkel

Oxidant signals and oxidative stress

Curr. Opin. Cell. Biol., 15 (2003), pp. 247-254

\section{[2] F.J. Giordano}

Oxygen, oxidative stress, hypoxia, and heart failure

J. Clin. Invest., 115 (2005), pp. 500-508

[3] J.D. Lambeth

NOX enzymes and the biology of reactive oxygen

Nat. Rev. Immunol., 4 (2004), pp. 181-189

[4] A.C. Cave, A.C. Brewer, A. Narayanapanicker, R. Ray, D.J. Grieve, S. Walker et al. NADPH oxidases in cardiovascular health and disease

Antioxid. Redox. Signal, 8 (2006), pp. 691-728

[5] K. Bedard, K.H. Krause

The NOX family of ROS-generating NADPH oxidases: physiology and pathophysiology

Physiol. Rev., 87 (2007), pp. 245-313

[6] B. Banfi, F. Tirone, I. Durussel, J. Knisz, P. Moskwa, G.Z. Molnar et al. Mechanism of $\mathrm{Ca} 2+$ activation of the NADPH oxidase 5 (NOX5)

J. Biol. Chem., 279 (2004), pp. 18583-18591

[7] B. Lassegue, D. Sorescu, K. Szocs, Q. Yin, M. Akers, Y. Zhang et al.

Novel gp91(phox) homologues in vascular smooth muscle cells: nox1 mediates angiotensin II-induced superoxide formation and redoxsensitive signaling pathways

Circ. Res., 88 (2001), pp. 888-894

[8] A. Gorlach, R.P. Brandes, K. Nguyen, M. Amidi, F. Dehghani, R. Busse

A gp91phox containing NADPH oxidase selectively expressed in endothelial cells is a major source of oxygen radical generation in the arterial wall

Circ. Res., 87 (2000), pp. 26-32 
Intracellular localization and preassembly of the NADPH oxidase complex in cultured endothelial cells

J. Biol. Chem., 277 (2002), pp. 19952-19960

[10] J.K. Bendall, A.C. Cave, C. Heymes, N. Gall, A.M. Shah

Pivotal role of a gp91(phox)-containing NADPH oxidase in angiotensin Il-induced cardiac hypertrophy in mice

Circulation, 105 (2002), pp. 293-296

[11] S.D. Hingtgen, X. Tian, J. Yang, S.M. Dunlay, A.S. Peek, Y. Wu et al.

Nox2-containing NADPH oxidase and Akt activation play a key role in angiotensin II-induced cardiomyocyte hypertrophy

Physiol. Genomics, 26 (2006), pp. 180-191

[12] L. Xiao, D.R. Pimentel, J. Wang, K. Singh, W.S. Colucci, D.B. Sawyer

Role of reactive oxygen species and $\mathrm{NAD}(\mathrm{P}) \mathrm{H}$ oxidase in alpha(1)-adrenoceptor signaling in adult rat cardiac myocytes

Am. J. Physiol. Cell. Physiol., 282 (2002), pp. C926-C934

[13] P.J. Pagano, J.K. Clark, M.E. Cifuentes-Pagano, S.M. Clark, G.M. Callis, M.T. Quinn

Localization of a constitutively active, phagocyte-like NADPH oxidase in rabbit aortic adventitia: enhancement by angiotensin II

Proc. Natl. Acad. Sci. U. S. A., 94 (1997), pp. 14483-14488

[14] R.M. Touyz, X. Chen, F. Tabet, G. Yao, G. He, M.T. Quinn et al.

Expression of a functionally active gp91phox-containing neutrophil-type NAD(P)H oxidase in smooth muscle cells from human resistance arteries: regulation by angiotensin II

Circ. Res., 90 (2002), pp. 1205-1213

[15] T. Ago, T. Kitazono, H. Ooboshi, T. Iyama, Y.H. Han, J. Takada et al.

Nox4 as the major catalytic component of an endothelial NAD(P)H oxidase

Circulation, 109 (2004), pp. 227-233

[16] L.L. Hilenski, R.E. Clempus, M.T. Quinn, J.D. Lambeth, K.K. Griendling

Distinct subcellular localizations of Nox1 and Nox4 in vascular smooth muscle cells

Arterioscler. Thromb. Vasc. Biol., 24 (2004), pp. 677-683

[17] J.A. Byrne, D.J. Grieve, J.K. Bendall, J.M. Li, C. Gove, J.D. Lambeth et al.

Contrasting roles of NADPH oxidase isoforms in pressure-overload versus angiotensin II-induced cardiac hypertrophy

Circ. Res., 93 (2003), pp. 802-805

[18] J. Li, M. Stouffs, L. Serrander, B. Banfi, E. Bettiol, Y. Charnay et al.

The NADPH oxidase NOX4 drives cardiac differentiation: role in regulating cardiac transcription factors and MAP kinase activation

Mol. Biol. Cell., 17 (2006), pp. 3978-3988

[19] I. Cucoranu, R. Clempus, A. Dikalova, P.J. Phelan, S. Ariyan, S. Dikalov et al.

NAD(P)H oxidase 4 mediates transforming growth factor-beta1-induced differentiation of cardiac fibroblasts into myofibroblasts

Circ. Res., 97 (2005), pp. 900-907

[20] R.S. BelAiba, T. Djordjevic, A. Petry, K. Diemer, S. Bonello, B. Banfi et al.

NOX5 variants are functionally active in endothelial cells

Free Radic. Biol. Med., 42 (2007), pp. 446-459

[21] D.B. Jay, C.A. Papaharalambus, B. Seidel-Rogol, A.E. Dikalova, B. Lassègue, K.K. Griendling

Nox5 mediates PDGF-induced proliferation in human aortic smooth muscle cells

Free Radic. Biol. Med., 45 (2008), pp. 329-335

[22] B. Lassegue, R.E. Clempus

Vascular NAD(P)H oxidases: specific features, expression, and regulation

Am. J. Physiol. Regul. Integr. Comp. Physiol., 285 (2003), pp. R277-R297

[23] R.E. Clempus, K.K. Griendling

Reactive oxygen species signaling in vascular smooth muscle cells

Cardiovasc. Res., 71 (2006), pp. 216-225 
[24] A. Narayana, A. Sirker, A.M. Shah

Redox sensitive signaling pathways in cardiac remodeling, hypertrophy and failure

Front. Biosci., 14 (2009), pp. 3168-3187

[25] R.P. Brandes

Role of NADPH oxidases in the control of vascular gene expression

Antioxid. Redox. Signal, 5 (2003), pp. 803-811

[26] R.S. Frey, A. Rahman, J.C. Kefer, R.D. Minshall, A.B. Malik

PKCzeta regulates TNF-alpha-induced activation of NADPH oxidase in endothelial cells

Circ. Res., 90 (2002), pp. 1012-1019

[27] J.M. Li, A.M. Shah

Mechanism of endothelial cell NADPH oxidase activation by angiotensin II. Role of the p47phox subunit

J. Biol. Chem., 278 (2003), pp. 12094-12100

[28] M. Ushio-Fukai, Y. Tang, T. Fukai, S.I. Dikalov, Y. Ma, M. Fujimoto et al.

Novel role of gp91(phox)-containing NAD(P)H oxidase in vascular endothelial growth factor-induced signaling and angiogenesis

Circ. Res., 91 (2002), pp. 1160-1167

[29] J.M. Li, A.M. Mullen, S. Yun, F. Wientjes, G.Y. Brouns, A.J. Thrasher et al.

Essential role of the NADPH oxidase subunit p47(phox) in endothelial cell superoxide production in response to phorbol ester and tumor necrosis factor-alpha

Circ. Res., 90 (2002), pp. 143-150

[30] M. Zhang, A.L. Kho, N. Anilkumar, R. Chibber, P.J. Pagano, A.M. Shah et al.

Glycated proteins stimulate reactive oxygen species production in cardiac myocytes: involvement of Nox2 (gp91phox)-containing NADPH oxidase

Circulation, 113 (2006), pp. 1235-1243

[31] F.J. Miller Jr., M. Filali, G.J. Huss, B. Stanic, A. Chamseddine, T.J. Barna et al.

Cytokine activation of nuclear factor kappa B in vascular smooth muscle cells requires signaling endosomes containing Nox 1 and ClC-3

Circ. Res., 101 (2007), pp. 663-671

[32] Q. Li, M.M. Harraz, W. Zhou, L.N. Zhang, W. Ding, Y. Zhang et al.

Nox2 and Rac1 regulate H2O2-dependent recruitment of TRAF6 to endosomal interleukin-1 receptor complexes

Mol. Cell. Biol., 26 (2006), pp. 140-154

[33] R.K. Ambasta, P. Kumar, K.K. Griendling, H.H. Schmidt, R. Busse, R.P. Brandes

Direct interaction of the novel Nox proteins with p22phox is required for the formation of a functionally active NADPH oxidase

J. Biol. Chem., 279 (2004), pp. 45935-45941

[34] K.D. Martyn, L.M. Frederick, K. von Loehneysen, M.C. Dinauer, U.G. Knaus

Functional analysis of Nox4 reveals unique characteristics compared to other NADPH oxidases

Cell. Signal, 18 (2006), pp. 69-82

[35] K. Chen, M.T. Kirber, H. Xiao, Y. Yang, J.F. Keaney Jr

Regulation of ROS signal transduction by NADPH oxidase 4 localization

J. Cell. Biol., 181 (2008), pp. 1129-1139

[36] K. Mahadev, H. Motoshima, X. Wu, J.M. Ruddy, R.S. Arnold, G. Cheng et al.

The NAD(P)H oxidase homolog Nox4 modulates insulin-stimulated generation of $\mathrm{H} 2 \mathrm{O} 2$ and plays an integral role in insulin signal transduction Mol. Cell. Biol., 24 (2004), pp. 1844-1854

[37] N. Anilkumar, R. Weber, M. Zhang, A. Brewer, A.M. Shah

Nox4 and nox2 NADPH oxidases mediate distinct cellular redox signaling responses to agonist stimulation

Arterioscler. Thromb. Vasc. Biol., 28 (2008), pp. 1347-1354

[38] S.A. Gupte

Glucose-6-phosphate dehydrogenase: a novel therapeutic target in cardiovascular diseases 
Curr. Opin. Investig. Drugs, 9 (2008), pp. 993-1000

[39] R. Dworakowski, N. Anilkumar, M. Zhang, A.M. Shah

Redox signaling involving NADPH oxidase-derived reactive oxygen species

Biochem. Soc. Trans., 34 (2006), pp. 960-964

[40] L.C. Hool, B. Corry

Redox control of calcium channels: from mechanisms to therapeutic opportunities

Antioxid. Redox. Signal, 9 (2007), pp. 409-435

[41] A.V. Zima, L.A. Blatter

Redox regulation of cardiac calcium channels and transporters

Cardiovasc. Res., 71 (2006), pp. 310-321

[42] G. Cherednichenko, A.V. Zima, W. Feng, S. Schaefer, L.A. Blatter, I.N. Pessah

NADH oxidase activity of rat cardiac sarcoplasmic reticulum regulates calcium-induced calcium release

Circ. Res., 94 (2004), pp. 478-486

[43] G. Sanchez, Z. Pedrozo, R.J. Domenech, C. Hidalgo, P. Donoso

Tachycardia increases NADPH oxidase activity and RyR2 S-glutathionylation in ventricular muscle

J. Mol. Cell. Cardiol., 39 (2005), pp. 982-991

[44] G. Sanchez, M. Escobar, Z. Pedrozo, P. Macho, R. Domenech, S. Hartel et al.

Exercise and tachycardia increase NADPH oxidase and ryanodine receptor-2 activity: possible role in cardioprotection

Cardiovasc. Res., 77 (2008), pp. 380-386

[45] X.Y. Yi, V.X. Li, F. Zhang, F. Yi, D.R. Matson, M.T. Jiang et al.

Characteristics and actions of $\mathrm{NAD}(\mathrm{P}) \mathrm{H}$ oxidase on the sarcoplasmic reticulum of coronary artery smooth muscle

Am. J. Physiol. Heart Circ. Physiol., 290 (2006), pp. H1136-H1144

[46] Q. Zeng, Q. Zhou, F. Yao, S.T. O'Rourke, C. Sun

Endothelin-1 regulates cardiac L-type calcium channels via NAD(P)H oxidase-derived superoxide

J. Pharmacol. Exp. Ther., 326 (2008), pp. 732-738

[47] Y. Shimoni, D. Hunt, M. Chuang, K.Y. Chen, G. Kargacin, D.L. Severson

Modulation of potassium currents by angiotensin and oxidative stress in cardiac cells from the diabetic rat

J. Physiol., 567 (2005), pp. 177-190

[48] Z. Ren, F.J. Raucci Jr., D.M. Browe, C.M. Baumgarten

Regulation of swelling-activated $\mathrm{Cl}(-)$ current by angiotensin II signaling and NADPH oxidase in rabbit ventricle

Cardiovasc. Res., 77 (2008), pp. 73-80

[49] C. Zhou, C. Ziegler, L.A. Birder, A.F. Stewart, E.S. Levitan

Angiotensin II and stretch activate NADPH oxidase to destabilize cardiac Kv4.3 channel mRNA

Circ. Res., 98 (2006), pp. 1040-1047

[50] C.T. Tsai, D.L. Wang, W.P. Chen, J.J. Hwang, C.S. Hsieh, K.L. Hsu et al.

Angiotensin II increases expression of alpha1C subunit of L-type calcium channel through a reactive oxygen species and cAMP response element-binding protein-dependent pathway in HL-1 myocytes

Circ. Res., 100 (2007), pp. 1476-1485

[51] L.L. Shang, S. Sanyal, A.E. Pfahnl, Z. Jiao, J. Allen, H. Liu et al.

NF-kappaB-dependent transcriptional regulation of the cardiac scn5a sodium channel by angiotensin II

Am. J. Physiol. Cell. Physiol., 294 (2008), pp. C372-C379

[52] J. Heineke, J.D. Molkentin

Regulation of cardiac hypertrophy by intracellular signaling pathways

Nat. Rev. Mol. Cell. Biol., 7 (2006), pp. 589-600

[53] K. Nakamura, K. Fushimi, H. Kouchi, K. Mihara, M. Miyazaki, T. Ohe et al. 
Inhibitory effects of antioxidants on neonatal rat cardiac myocyte hypertrophy induced by tumor necrosis factor-alpha and angiotensin II Circulation, 98 (1998), pp. 794-799

[54] S. Hirotani, K. Otsu, K. Nishida, Y. Higuchi, T. Morita, H. Nakayama et al.

Involvement of nuclear factor-kappaB and apoptosis signal-regulating kinase 1 in G-protein-coupled receptor agonist-induced cardiomyocyte hypertrophy

Circulation, 105 (2002), pp. 509-515

[55] J.M. Li, N.P. Gall, D.J. Grieve, M. Chen, A.M. Shah

Activation of NADPH oxidase during progression of cardiac hypertrophy to failure

Hypertension, 40 (2002), pp. 477-484

[56] C. Heymes, J.K. Bendall, P. Ratajczak, A.C. Cave, J.L. Samuel, G. Hasenfuss et al. Increased myocardial NADPH oxidase activity in human heart failure

J. Am. Coll. Cardiol., 41 (2003), pp. 2164-2171

[57] C. Maack, T. Kartes, H. Kilter, H.J. Schafers, G. Nickenig, M. Bohm et al.

Oxygen free radical release in human failing myocardium is associated with increased activity of rac1-GTPase and represents a target for statin treatment

Circulation, 108 (2003), pp. 1567-1574

[58] C. Nediani, E. Borchi, C. Giordano, S. Baruzzo, V. Ponziani, M. Sebastiani et al.

NADPH oxidase-dependent redox signaling in human heart failure: relationship between the left and right ventricle

J. Mol. Cell. Cardiol., 42 (2007), pp. 826-834

[59] M. Satoh, H. Ogita, K. Takeshita, Y. Mukai, D.J. Kwiatkowski, J.K. Liao

Requirement of Rac1 in the development of cardiac hypertrophy

Proc. Natl. Acad. Sci. U. S. A., 103 (2006), pp. 7432-7437

[60] M.A. Sussman, S. Welch, A. Walker, R. Klevitsky, T.E. Hewett, R.L. Price et al.

Altered focal adhesion regulation correlates with cardiomyopathy in mice expressing constitutively active rac1

J. Clin. Invest., 105 (2000), pp. 875-886

[61] J.B. Pracyk, K. Tanaka, D.D. Hegland, K.S. Kim, R. Sethi, Rovira et al.

A requirement for the rac1 GTPase in the signal transduction pathway leading to cardiac myocyte hypertrophy

J. Clin. Invest., 102 (1998), pp. 929-937

[62] Y. Higuchi, K. Otsu, K. Nishida, S. Hirotani, H. Nakayama, O. Yamaguchi et al.

The small GTP-binding protein Rac1 induces cardiac myocyte hypertrophy through the activation of apoptosis signal-regulating kinase 1 and nuclear factor-kappa B

J. Biol. Chem., 278 (2003), pp. 20770-20777

[63] M. Maytin, D.A. Siwik, M. Ito, L. Xiao, D.B. Sawyer, R. Liao et al.

Pressure overload-induced myocardial hypertrophy in mice does not require gp91phox

Circulation, 109 (2004), pp. 1168-1171

[64] L. Xiao, D.R. Pimental, J.K. Amin, K. Singh, D.B. Sawyer, W.S. Colucci

MEK1/2-ERK1/2 mediates alpha1-adrenergic receptor-stimulated hypertrophy in adult rat ventricular myocytes

J. Mol. Cell. Cardiol., 33 (2001), pp. 779-787

[65] G.M. Kuster, D.R. Pimentel, T. Adachi, Y. Ido, D.A. Brenner, R.A. Cohen et al.

Alpha-adrenergic receptor-stimulated hypertrophy in adult rat ventricular myocytes is mediated via thioredoxin-1-sensitive oxidative modification of thiols on Ras

Circulation, 111 (2005), pp. 1192-1198

[66] H. Nakagami, M. Takemoto, J.K. Liao

NADPH oxidase-derived superoxide anion mediates angiotensin II-induced cardiac hypertrophy

J. Mol. Cell. Cardiol., 35 (2003), pp. 851-859

[67] Y. Izumiya, S. Kim, Y. Izumi, K. Yoshida, M. Yoshiyama, A. Matsuzawa et al. 
Apoptosis signal-regulating kinase 1 plays a pivotal role in angiotensin II-induced cardiac hypertrophy and remodeling

Circ. Res., 93 (2003), pp. 874-883

[68] A. Matsuzawa, H. Ichijo

Stress-responsive protein kinases in redox-regulated apoptosis signaling

Antioxid. Redox. Signal, 7 (2005), pp. 472-481

[69] A. Gonzalez, S. Ravassa, B. Lopez, I. Loperena, R. Querejeta, J. Diez

Apoptosis in hypertensive heart disease: a clinical approach

Curr. Opin. Cardiol., 21 (2006), pp. 288-294

[70] M.T. Crow, K. Mani, Y.J. Nam, R.N. Kitsis

The mitochondrial death pathway and cardiac myocyte apoptosis

Circ. Res., 95 (2004), pp. 957-970

[71] F. Qin, R. Patel, C. Yan, W. Liu

NADPH oxidase is involved in angiotensin II-induced apoptosis in H9C2 cardiac muscle cells: effects of apocynin

Free Radic. Biol. Med., 40 (2006), pp. 236-246

[72] F. Qin, M. Simeone, R. Patel

Inhibition of NADPH oxidase reduces myocardial oxidative stress and apoptosis and improves cardiac function in heart failure after myocardial infarction

Free Radic. Biol. Med., 43 (2007), pp. 271-281

[73] A. Dandapat, C.P. Hu, D. Li, Y. Liu, H. Chen, P.L. Hermonat et al.

Overexpression of TGFbeta1 by adeno-associated virus type-2 vector protects myocardium from ischemia-reperfusion injury

Gene. Ther., 15 (2008), pp. 415-423

[74] V. Grishko, V. Pastukh, V. Solodushko, M. Gillespie, J. Azuma, S. Schaffer

Apoptotic cascade initiated by angiotensin II in neonatal cardiomyocytes: role of DNA damage

Am. J. Physiol. Heart Circ. Physiol., 285 (2003), pp. H2364-H2372

[75] S. Li, X. Jiao, L. Tao, H. Liu, Y. Cao, B.L. Lopez et al.

Tumor necrosis factor-alpha in mechanic trauma plasma mediates cardiomyocyte apoptosis

Am. J. Physiol. Heart Circ. Physiol., 293 (2007), pp. H1847-H1852

[76] H. Hayashi, M. Kobara, M. Abe, N. Tanaka, E. Gouda, H. Toba et al.

Aldosterone nongenomically produces NADPH oxidase-dependent reactive oxygen species and induces myocyte apoptosis

Hypertens. Res., 31 (2008), pp. 363-375

[77] N.S. Dhalla, R.M. Temsah, T. Netticadan

Role of oxidative stress in cardiovascular diseases

J. Hypertens., 18 (2000), pp. 655-673

[78] R.A. Josephson, H.S. Silverman, E.G. Lakatta, M.D. Stern, J.L. Zweier

Study of the mechanisms of hydrogen peroxide and hydroxyl free radical-induced cellular injury and calcium overload in cardiac myocytes

J. Biol. Chem., 266 (1991), pp. 2354-2361

[79] W.D. Gao, Y. Liu, E. Marban

Selective effects of oxygen free radicals on excitation-contraction coupling in ventricular muscle. Implications for the mechanism of stunned myocardium

Circulation, 94 (1996), pp. 2597-2604

[80] Y.W. Xie, P.M. Kaminski, M.S. Wolin

Inhibition of rat cardiac muscle contraction and mitochondrial respiration by endogenous peroxynitrite formation during posthypoxic reoxygenation

Circ. Res., 82 (1998), pp. 891-897

[81] S. Kinugawa, H. Tsutsui, S. Hayashidani, T. Ide, N. Suematsu, S. Satoh et al. 
Treatment with dimethylthiourea prevents left ventricular remodeling and failure after experimental myocardial infarction in mice: role of oxidative stress

Circ. Res., 87 (2000), pp. 392-398

[82] T. Ide, H. Tsutsui, S. Hayashidani, D. Kang, N. Suematsu, K. Nakamura et al.

Mitochondrial DNA damage and dysfunction associated with oxidative stress in failing hearts after myocardial infarction

Circ. Res., 88 (2001), pp. 529-535

[83] P. Kaplan, E. Babusikova, J. Lehotsky, D. Dobrota

Free radical-induced protein modification and inhibition of Ca2+-ATPase of cardiac sarcoplasmic reticulum

Mol. Cell. Biochem., 248 (2003), pp. 41-47

[84] M. Yano, S. Okuda, T. Oda, T. Tokuhisa, H. Tateishi, M. Mochizuki et al.

Correction of defective interdomain interaction within ryanodine receptor by antioxidant is a new therapeutic strategy against heart failure Circulation, 112 (2005), pp. 3633-3643

[85] D.J. Grieve, J.A. Byrne, A. Siva, J. Layland, S. Johar, A.C. Cave et al.

Involvement of the nicotinamide adenosine dinucleotide phosphate oxidase isoform Nox 2 in cardiac contractile dysfunction occurring in response to pressure overload

J. Am. Coll. Cardiol., 47 (2006), pp. 817-826

[86] S.Y. Li, X. Yang, A.F. Ceylan-Isik, M. Du, N. Sreejayan, J. Ren

Cardiac contractile dysfunction in Lep/Lep obesity is accompanied by NADPH oxidase activation, oxidative modification of sarco(endo)plasmic reticulum Ca2+-ATPase and myosin heavy chain isozyme switch

Diabetologia, 49 (2006), pp. 1434-1446

[87] L.E. Wold, A.F. Ceylan-Isik, C.X. Fang, X. Yang, S.Y. Li, N. Sreejayan et al.

Metallothionein alleviates cardiac dysfunction in streptozotocin-induced diabetes: role of Ca2+ cycling proteins, NADPH oxidase, poly(ADP-

Ribose) polymerase and myosin heavy chain isozyme

Free Radic. Biol. Med., 40 (2006), pp. 1419-1429

[88] Z. Guo, Z. Xia, J. Jiang, J.H. McNeill

Downregulation of NADPH oxidase, antioxidant enzymes, and inflammatory markers in the heart of streptozotocin-induced diabetic rats by Nacetyl-I-cysteine

Am. J. Physiol. Heart Circ. Physiol., 292 (2007), pp. H1728-H1736

[89] X. Yang, T.A. Doser, C.X. Fang, J.M. Nunn, R. Janardhanan, M. Zhu et al.

Metallothionein prolongs survival and antagonizes senescence-associated cardiomyocyte diastolic dysfunction: role of oxidative stress

FASEB J., 20 (2006), pp. 1024-1026

[90] T. Peng, X. Lu, Q. Feng

Pivotal role of gp91phox-containing NADH oxidase in lipopolysaccharide-induced tumor necrosis factor-alpha expression and myocardial depression

Circulation, 111 (2005), pp. 1637-1644

[91] P.A. MacCarthy, D.J. Grieve, J.M. Li, C. Dunster, F.J. Kelly, A.M. Shah

Impaired endothelial regulation of ventricular relaxation in cardiac hypertrophy: role of reactive oxygen species and NADPH oxidase Circulation, 104 (2001), pp. 2967-2974

[92] S. Johar, A.C. Cave, A. Narayanapanicker, D.J. Grieve, A.M. Shah

Aldosterone mediates angiotensin II-induced interstitial cardiac fibrosis via a Nox2-containing NADPH oxidase

FASEB J., 20 (2006), pp. 1546-1548

[93] Y. Sun, J. Zhang, L. Lu, S.S. Chen, M.T. Quinn, K.T. Weber

Aldosterone-induced inflammation in the rat heart: role of oxidative stress

Am. J. Pathol., 161 (2002), pp. 1773-1781

[94] Y.M. Park, M.Y. Park, Y.L. Suh, J.B. Park

NAD(P)H oxidase inhibitor prevents blood pressure elevation and cardiovascular hypertrophy in aldosterone-infused rats

Biochem. Biophys. Res. Commun., 313 (2004), pp. 812-817 
[95] A. Whaley-Connell, G. Govindarajan, J. Habibi, M.R. Hayden, S.A. Cooper, Y. Wei et al.

Angiotensin II-mediated oxidative stress promotes myocardial tissue remodeling in the transgenic (mRen2) 27 Ren2 rat

Am. J. Physiol. Endocrinol. Metab., 293 (2007), pp. E355-E363

[96] K. Grote, I. Flach, M. Luchtefeld, E. Akin, S.M. Holland, H. Drexler et al.

Mechanical stretch enhances mRNA expression and proenzyme release of matrix metalloproteinase-2 (MMP-2) via NAD(P)H oxidase-derived reactive oxygen species

Circ. Res., 92 (2003), pp. e80-e86

[97] M. Luchtefeld, K. Grote, C. Grothusen, S. Bley, N. Bandlow, T. Selle et al.

Angiotensin II induces MMP-2 in a p47phox-dependent manner

Biochem. Biophys. Res. Commun., 328 (2005), pp. 183-188

[98] N.G. Frangogiannis

Targeting the inflammatory response in healing myocardial infarcts

Curr. Med. Chem., 13 (2006), pp. 1877-1893

[99] D.J. Grieve, J.A. Byrne, A.C. Cave, A.M. Shah

Role of oxidative stress in cardiac remodelling after myocardial infarction

Heart Lung Circ., 13 (2004), pp. 132-138

[100] M.F. Hill, P.K. Singal

Antioxidant and oxidative stress changes during heart failure subsequent to myocardial infarction in rats

Am. J. Pathol., 148 (1996), pp. 291-300

[101] Y.T. Sia, N. Lapointe, T.G. Parker, J.N. Tsoporis, C.F. Deschepper, A. Calderone et al. Beneficial effects of long-term use of the antioxidant probucol in heart failure in the rat

Circulation, 105 (2002), pp. 2549-2555

[102] T. Fukui, M. Yoshiyama, A. Hanatani, T. Omura, J. Yoshikawa, Y. Abe

Expression of p22-phox and gp91-phox, essential components of NADPH oxidase, increases after myocardial infarction

Biochem. Biophys. Res. Commun., 281 (2001), pp. 1200-1206

[103] P.A. Krijnen, C. Meischl, C.E. Hack, C.J. Meijer, C.A. Visser, D. Roos et al.

Increased Nox2 expression in human cardiomyocytes after acute myocardial infarction

J. Clin. Pathol., 56 (2003), pp. 194-199

[104] C. Doerries, K. Grote, D. Hilfiker-Kleiner, M. Luchtefeld, A. Schaefer, S.M. Holland et al.

Critical role of the $\mathrm{NAD}(\mathrm{P}) \mathrm{H}$ oxidase subunit p47phox for left ventricular remodeling/dysfunction and survival after myocardial infarction

Circ. Res., 100 (2007), pp. 894-903

[105] Y.H. Looi, D.J. Grieve, A. Siva, S.J. Walker, N. Anilkumar, A.C. Cave et al.

Involvement of Nox2 NADPH oxidase in adverse cardiac remodeling after myocardial infarction

Hypertension, 51 (2008), pp. 319-325

[106] D.M. Yellon, J.M. Downey

Preconditioning the myocardium: from cellular physiology to clinical cardiology

Physiol. Rev., 83 (2003), pp. 1113-1151

[107] R.A. Forbes, C. Steenbergen, E. Murphy

Diazoxide-induced cardioprotection requires signaling through a redox-sensitive mechanism

Circ. Res., 88 (2001), pp. 802-809

[108] T. Pain, X.M. Yang, S.D. Critz, Y. Yue, A. Nakano, G.S. Liu et al.

Opening of mitochondrial K(ATP) channels triggers the preconditioned state by generating free radicals

Circ. Res., 87 (2000), pp. 460-466

[109] D.K. Das, N. Maulik

Preconditioning potentiates redox signaling and converts death signal into survival signal 
Arch. Biochem. Biophys., 420 (2003), pp. 305-311

[110] R.M. Bell, A.C. Cave, S. Johar, D.J. Hearse, A.M. Shah, M.J. Shattock

Pivotal role of NOX-2-containing NADPH oxidase in early ischemic preconditioning

FASEB J., 19 (2005), pp. 2037-2039

[111] S. Kimura, G.X. Zhang, A. Nishiyama, T. Shokoji, L. Yao, Y.Y. Fan et al.

Role of $\mathrm{NAD}(\mathrm{P}) \mathrm{H}$ oxidase- and mitochondria-derived reactive oxygen species in cardioprotection of ischemic reperfusion injury by angiotensin II Hypertension, 45 (2005), pp. 860-866

[112] S. Das, R.M. Engelman, N. Maulik, D.K. Das

Angiotensin preconditioning of the heart: evidence for redox signaling

Cell. Biochem. Biophys., 44 (2006), pp. 103-110 